\title{
Effective Energy Constraint Routing with on-Demand Routing Protocols in MANET
}

\author{
Kanwal Deep Kaur, Manish Bhardwaj \\ Department of Computer science and Engineering, SRM University, NCR Campus, Modinagar, Ghaziabad, India
}

Email address:

kanwaldp92@gmail.com (K. D. Kaur), aapkaapna13@gmail.com (M. Bhardwaj)

To cite this article:

Kanwal Deep Kaur, Manish Bhardwaj. Effective Energy Constraint Routing with On-Demand Routing Protocols in MANET. American Journal of Networks and Communications. Vol. 4, No. 2, 2015, pp. 21-24. doi: 10.11648/j.ajnc.20150402.12

\begin{abstract}
MANET is a dynamically reconfigurable wireless network with no fixed infrastructure. Each node acts as a router and host and it moves in an arbitrary manner in many ad hoc networks. It is significant that an energy conscious routing strategy should be adopted to minimize the energy required for communication. In such an environment, each host acts as a router and forwards packets to the next hop in order to reach, hop by hop, the final target. The major requirement in the MANET is which are usually characterized by mobile nodes with limited battery, is to limit the energy consumption.So, in this paper we are going to do the effective routing with the on demand protocols. The proposed method will try to prove that RAODV is better protocol than AODV and DSR using Network Simulator. To meet the new challenges, innovative protocols are needed to achieve energy efficiency, flexible scalability and adaptability and good network performance. This paper seeks to study and analyze the on demand routing protocols with identical loads and environment conditions and also evaluate their relative performance with respect to performance metrics.
\end{abstract}

Keywords: Mobile Ad-hoc Network, MANET, AODV, DSR, RAODV

\section{Introduction}

A mobile ad hoc network (MANET) is a collection of wireless mobile hosts which cooperatively forms a network. It is continuously, self-configuring, infrastructure-less network of mobile devices connected without wires. It uses no fixed infrastructure or centralized administration. Each device in a MANET is free to move independently in any direction and perform routing function to establish communication. Due to this it can change its links to other devices frequently. MANET requires that each device continuously maintain the information required to properly route traffic. In Manet's death of even a few of the nodes due to power exhaustion might cause disconnect of services. MANETs are a kind of Wireless ad hoc network that usually has a routable networking environment. Many academic papers evaluate protocols and their abilities on the basis of varying degrees of mobility and other parameters. Different protocols are then evaluated based on certain performance measures such as the packet drop rate, overhead, end-to-end packet delays, network throughput, ability to scale, etc. As we know that in Mobile Ad-Hoc Networks, mobile devices are equipped with power, so many energy aware routing strategies should be adopted to utilize this energy equipped devices resourcefully for transmission of data packets. On the basis of these routing strategies, the energy aware performance metrics are analyzed on routing protocols like Ad-Hoc on Demand Distance Vector Protocol (AODV), Distance Source Routing Protocol (DSR) and Reverse Ad-Hoc on Demand Distance Vector Protocol (RAODV). The comparison results prove that RAODV protocol reduces the energy consumption and can be adopted for any routing strategy, in order to increase the performance of the network lifetime in comparison with DSR and AODV.

This paper is organized as follows: Section 1 consists of the Introduction part; while Section 2 provides a brief summary of existing protocols. In Section 3 the simulation model and the performance metrics required have been discussed. The experimental results have been discussed in Section 4.Section 5 concludes our work as well as findings.

\section{Routing Protocols}

Numerous routing protocols have been developed for ad hoc mobile networks. Routing in MANETs can be done in 
many ways. On the basis of the routing strategy the routing protocols can be categorized as Table-Driven and On-Demand Protocols.

I. Table-driven routing protocols: In this routing table is maintained at each node each node. The routing table contains the routing information to every other node in the network. All nodes maintain an update table for a consistent and up-to-date view of the network. The routing procedure is based on the information in these tables.

II. On-Demand Routing Protocols: These protocols differ from table-driven routing protocols. In this the routes are created as and when required instead of maintaining up-to-date routes at every node. We can say that they take a lazy approach. It invokes the route discovery mechanisms to find the path from source to destination.

There are several well known routing protocols exist, among them we are discussing some basic one which are somehow related to our work:

A. AODV

AODV is a routing protocol for MANETs and other wireless adhoc networks. In AODV Routing Protocol a route is established only when it is required by a source node for transmitting data packets. AODV builds routes using a route request and route reply mechanism. When a source node desires a route to a destination it broadcasts a route request (RREQ) packet across the network. Nodes receiving this packet update their information for the source node. The RREQ contains the most recent sequence number, source node's IP address, sequence number and broadcast ID. If the node that receives RREQ is either a destination or if it has route to destination with corresponding sequence number it sends a route reply i.e. RREP. In this case, it unicasts a RREP back to the source; otherwise, it rebroadcasts the RREQ. If nodes receive a RREQ which is already processed, the node discards the RREQ and does not forward it. As the RREP propagates back to the source nodes set up forward pointers to the destination. After receiving the RREP the source node may begin to forward data packets to the destination.

\section{B. DSR}

DSR is a reactive routing protocol for MANETs. It forms a route on demand when a node requests it.DSR uses a source routing approach. It has two phases route discovery and route maintenance. Both phases work together to allow nodes to discover and maintain source routes to destinations. When a source node wants to find a route, the source node initiates a route discovery process. It broadcasts a Route Request (RREQ) to the entire network. The RREQ is forwarded till either the destination is reached or another node is found with a route to the destination. Each node appends own identifier when forwarding RREQ. After destination node received the first RREQ it sends RREP by reversing the route. It is similar to AODV but it uses source routing where all routing information is maintained at mobile nodes. For determining routes it is necessary to accumulate the address of each device between the source and destination during route discovery. The learned paths are used to route packets. To accomplish source routing, the routed packets contain the address of each device the packet will traverse. When route error packets are generated the route maintenance phase is initiated and it removes the erroneous hop from node's cache.

\section{RAODV}

RAODV is the on demand routing protocol that discovers routes by using reverse route discovery procedure. Most of the on demand ad hoc routing protocols use single route reply along the reverse path. In many routing protocols there is disadvantage of increase in power consumption and communication delay. Packet delivery ratio is another disadvantage of using many other routing protocols. To avoid these problems, a Reverse Adhoc On demand Distance Vector routing protocol (RAODV) has been approached. Due to rapid change of topology the RREP could not arrive to the source node. In case of high mobility, after a source node sends several RREQ packets; the node obtains a reply packet. To reduces the path fail correction packets and to obtain better performance than other protocols RAODV tries multiple route replies.

During route discovery procedure, source node and destination node plays the same role from the point of sending control packets. After receiving RREQ, destination node floods R-RREQ to find the source node. Since $\mathrm{R}-\mathrm{AODV}$ is reactive routing protocol, no permanent routes are stored in nodes. Route discovery procedure is initiated by broadcasting by the source node. The RREQ message contains the information about source address, destination address, broadcast ID, hop count, message type, source sequence number, destination sequence number, request time. Thus, after receiving RREQ message, destination node floods reverse route request (R-RREQ), to find source node. When source node receives an R-RREQ message, data packet transmission is started immediately.

\section{Simulation Model}

A discrete Network Simulator NS2 2.35 was used for the simulation purpose.

Table 1. Simulation model

\begin{tabular}{ll}
\hline SIMULATOR & Network Simulator 2 \\
\hline NUMBER OF NODES & Random \\
TOPOLOGY & Random \\
INTERFACE TYPE & Phy/WirelessPhy \\
MAC TYPE & 802.11 \\
QUEUE TYPE & Droptail/Priority Queue \\
QUEUE LENGTH & 50 Packets \\
ANTENNA TYPE & Omni Antenna \\
PROPAGATION TYPE & TwoRayGround \\
ROUTING PROTOCOL & AODV, DSR,RAODV \\
TRANSPORT AGENT & UDP \\
APPLICATION AGENT & CBR \\
INITIAL ENERGY & 100Joules \\
SIMULATION TIME & 10seconds \\
\hline
\end{tabular}

The different parameters considered for simulation are listed below:

A. METRICS ANALYSED FOR SIMULATION

Consumed Energy The number of nodes in the network 
versus the total consumed energy is considered as a metric.

Packet Delivery Ratio PDR is the proportion to the total amount of packets reached the receiver and amount of packet sent by source. If the amount of malicious node increases, PDR decreases. The higher mobility of nodes causes PDR to decrease.

$$
P D R(\%)=\frac{\text { Number of packets successfully delivered }}{\text { Number of packets generated }}
$$

Throughput The amount of data successfully received at the destination.

$$
\text { Throughput }(\text { bits } / s)=\frac{\text { Total Data }}{\text { Data Transmission duration }}
$$

Routing Overhead It is the number of routing packets transmitted per data packet delivered to the destination.

Remaining Energy It refers to the remaining energy available in the node after data transmission.

\section{Experimental Results}

The following graphical analysis shows the performance results of AODV, DSR and RAODV.

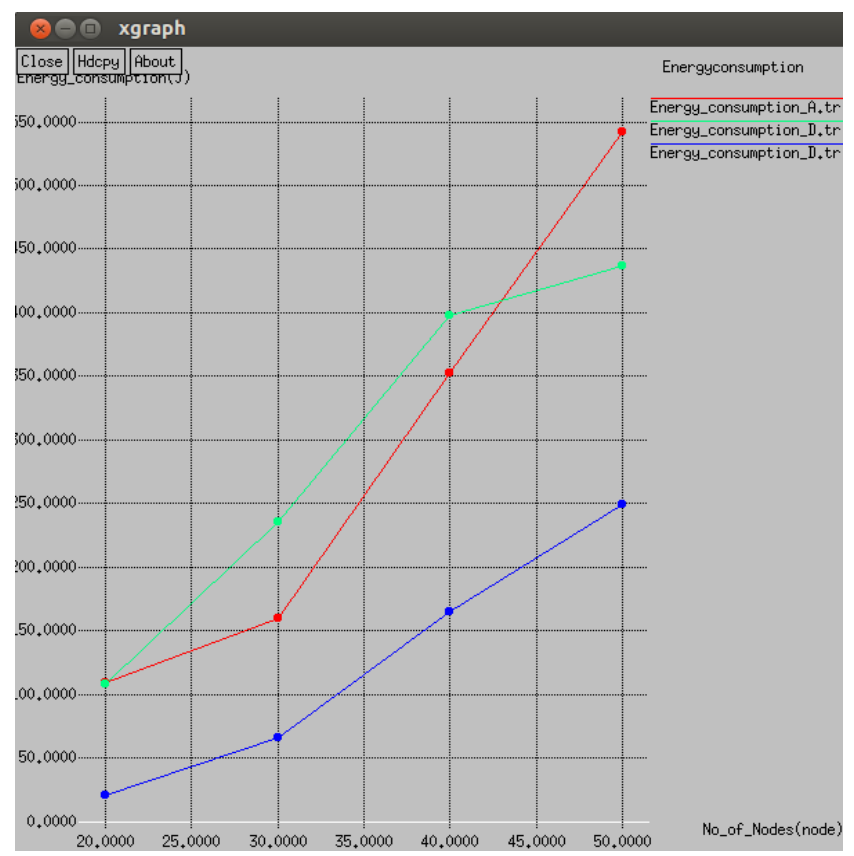

Figure 1. Number of nodes vs consumed energy.

When the numbers of nodes are increased Energy consumption of the network is increased. In Fig 1, when comparing to DSR and AODV protocol RAODV consumes less energy for data transmission.DSR and AODV needs more amount of energy for data transmission.

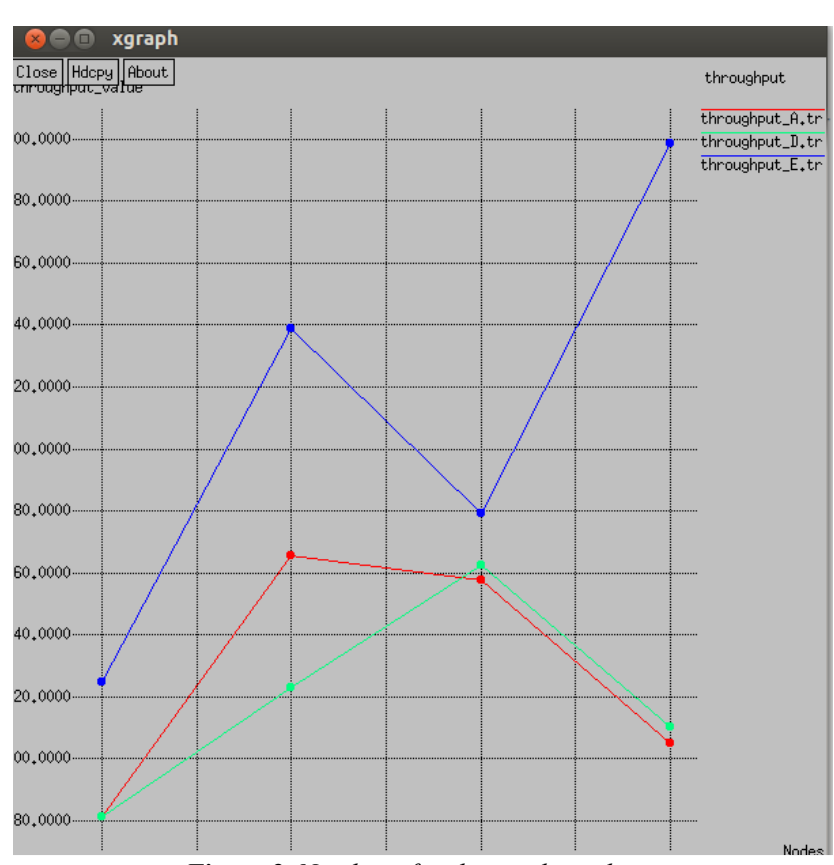

Figure 2. Number of nodes vs. throughput.

When the number of nodes are increased the network traffic is increased, packet delay is increased and packets are losses, collision occurred so the throughput value is reduced. Fig 2, shows the performance of different protocols where RAODV has high average rate of successful message delivery. RAODV will increase the network lifetime but in between the performance goes down because more number of packets are dropped due to link failure. Throughput in DSR is lesser than the throughput in AODV and RAODV.

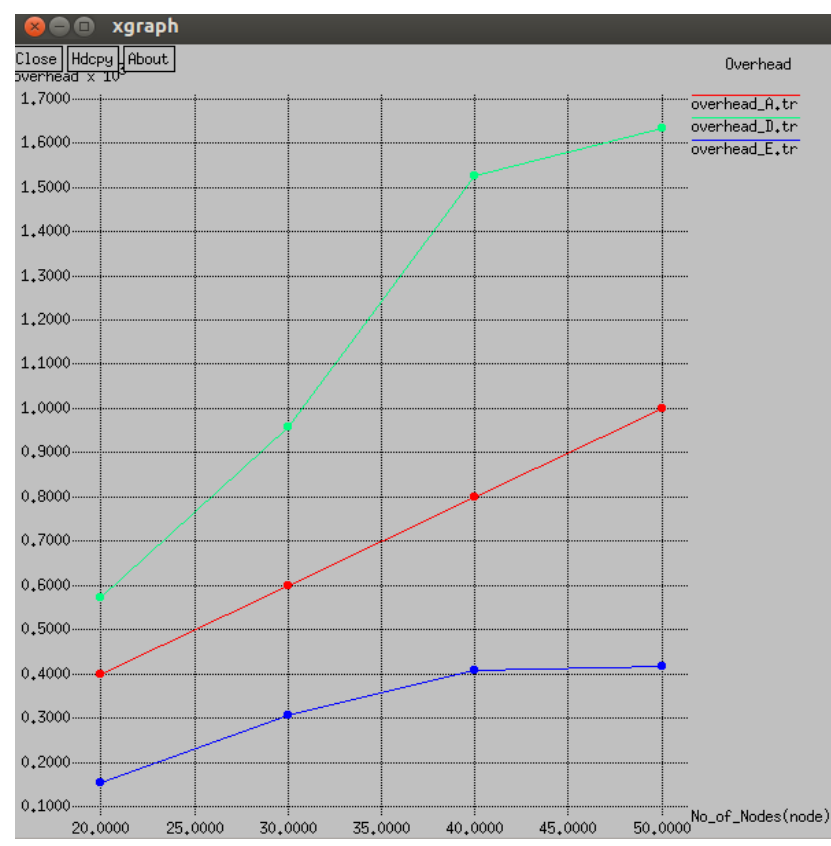

Figure 3. Number of nodes vs. overhead.

When the numbers of nodes are increased overhead is increased. Fig 3 shows overhead in DSR are greater than the overhead in AODV and RAODV. 


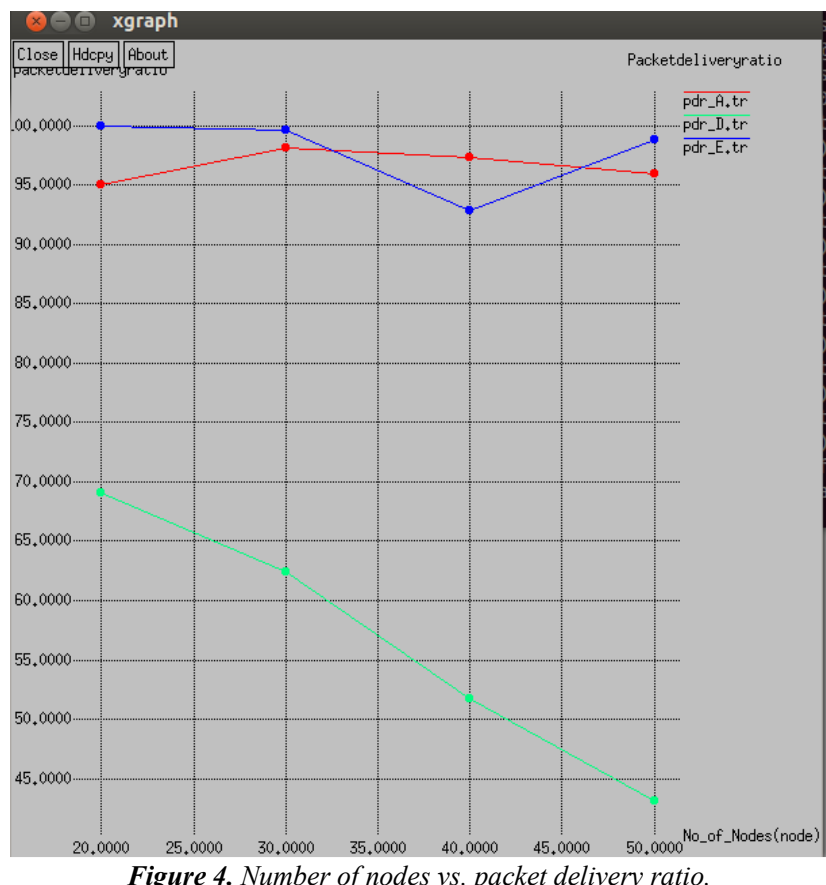

Figure 4. Number of nodes vs. packet delivery ratio.

Fig 4 comprehends that RAODV produces more PDR when comparing to DSR and AODV because RAODV can send and receive more number of packets. When the number of nodes is increased the packet delivery ratio is decreased. Packet delivery ratio in DSR are lesser than the packet delivery ratio in AODV and RAODV.

\section{Conclusion}

The various energy-aware parameters are analyzed in NS 2.35 for AODV, DSR and RAODV protocol by increasing the density of nodes. It is concluded that, RAODV performs well in Packet Delivery Ratio (PDR) but in some situations due to link breakage PDR is low.RAODV can send more number of packets as compared to DSR and AODV.Based on energy consumption DSR performs low in state compared with AODV and RAODV. Overhead in DSR is greater than the overhead in AODV and RAODV. RAODV will increase the network lifetime but in between the performance goes down because more number of packets are dropped due to link failure. Throughput in DSR is lesser than the throughput in AODV and RAODV.

\section{References}

[1] Manish Bhardwaj, Selection of Efficient Relay for Energy-Efficient Cooperative Ad Hoc Networks, American Journal of Networks and Communications. Special Issue:Ad Hoc Networks. Vol. 4, No. 3-1, 2015, pp. 5-11. doi: 10.11648/j.ajnc.s.2015040301.12.

[2] Manish Bhardwaj, C2 Torus New Interconnection Network Topology Based on 2D Torus, American Journal of Networks and Communications. Special Issue:Ad Hoc Networks. Vol. 4, No. 3-1, 2015, pp. 1-4. doi: 10.11648/j.ajnc.s.2015040301.11

[3] Bhabani Sankar Gouda, Ashish Kumar Das, K.Lakshmi Narayana "A Comparative Performance Analysis Of Energy Efficient Routing Protocols In Different Traffic Based Mobile Adhoc Networks",IEEE,2013.

[4] Anu Kumari, Arvind Kumar, Akhil Sharma "Survey Paper on Energy Efficient Routing Protocol in MANET", in IJARCSSE, vol. 3,2013 .

[5] Bhabani Sankar Gouda, Arkaprava Bhaduri, K.Lakshmi Narayana"Simulation and Comparative Analysis of Energy Conservation Performance Metric For ERAODV,AODV and DSDV Routing Protocols In MANETs",IEEE,2012.

[6] Zhimu Huang, Ryo Yamamoto, Yoshiaki Tanaka, "A Multipath Energy-Efficient Probability Routing Protocol in Ad Hoc Networks" ICACT 2014.

[7] Abdul Hadi Abd Rahman, Zuriati Ahmad Zukarnain"Performance Comparison of AODV, DSDV and I-DSDVmRouting Protocols in Mobile Ad Hoc Networks" European Journal of Scientific Research ISSN 1450-216X Vol.31 No.4 (2009), pp.566-576.

[8] Ms. Sujata Wasudeorao Wankhade, Prof. Dr. P. R. Deshmukh"Comparison of AODV and RAODV Routing Protocols in Mobile Ad Hoc Networks" IJARCSSE Volume 3, Issue 1, January 2013.

[9] C. Yu, B. Lee, Hee Yong Youn"Energy Efficient Routing Protocols for Mobile Ad Hoc Networks", Cleveland State University, EFFRD Grant No. 0210-0630-10.

[10] David B. Johnson, David A. Maltz,” Dynamic Source Routing in Ad Hoc Wireless Networks" (Kluwer Academic, 1996).

[11] Priti Garg, Asma Tuteja, "Comparative Performance Analysis of Two Ad-hoc Routing Protocols" IPCSIT vol.11 (2011).

[12] S.Shah, Amit Khandre, Mahesh Shirole and Girish Bhole," Performance Evaluation of Ad Hoc Routing Protocols Using NS2 Simulation," Proceeding of the National Conference on Mobile and pervasive Computing (CoMPC-2008), Chennai, India, August 2008.

[13] Anurag Malik, "Performance Analysis of routing protocol in Mobile Ad Hoc Network using NS 2" MIT International Journal of Computer Science \& Information Technology Vol. 1 No. 1 Jan 2011.

[14] V. Kanakaris, D. Ndzi and D. Azzi” Ad-hoc Networks Energy Consumption: A review of the Ad-Hoc Routing Protocols "Journal of Engineering Science and Technology Review 3 (1) (2010).

[15] Rishiwal, Mano Yadav, S. Verma, S. K. Bajapai "Power Aware Routing in Ad Hoc Wireless Networks", Journal of Computer Science and Technology, vol. 9, no. 2, pp. 101-109, October 2009.

[16] Getsy S Sara, Neelavathy Pari.S, Sridharan.D"Energy Efficient Ad Hoc On Demand Multipath Distance Vector Routing Protocol" International Journal of Recent Trends in Engineering, Vol 2, No. 3, November 2009. 